\title{
Viability of saliva and sputum samples from exacerbations in COPD patients with a metabolomic signature
}

\author{
Asibey-Berko T.1, Lewis K. ${ }^{1,2}$, Mur L. ${ }^{3}$, Mironas A. ${ }^{3}$, Asandei D.R. ${ }^{3}$, Ghosh S. ${ }^{1}$ \\ ${ }^{1}$ Hywel Dda University Health Board, Wales, U.K., ${ }^{2}$ School of Medicine, Swansea University, Wales, UK, \\ ${ }^{3}$ Institute of Biological, Environmental and Rural Sciences (IBERS), Aberystwyth University, Aberystwyth, Wales, U.K.
}

\section{Background:}

Chronic obstructive pulmonary disease (COPD) is the third leading cause of mortality worldwide. Exacerbations of COPD (ECOPD) are associated with increased mortality, worsening quality of life and high healthcare utilisation. ECOPD are associated with changes in bronchial inflammatory status. Our pilot studies have identified and patented a number of novel metabolite biomarkers in sputum which correlate with severity of airways obstruction. The metabolome represents the summation of genetic and protein mediated events and may be considered the most discriminatory (Fig. 1). We studied the reliability of changes in these biomarkers to predict ECOPD for timely treatment of exacerbations and pre-operative optimization. The challenge now is to translate this new information into clinical practice through the development of smarter and more accurate tests by integrating advancements in molecular biology with clinical trials from bench to bedside.

\section{Objective:}

To identify potential biomarkers for ECOPD by comparing concentrations of various metabolites in saliva and sputum samples from patients suffering from ECOPD, patients with stable COPD and healthy CONTROLS.

\section{Materials and Methods:}

Following ethics approval, the study was registered ISRCTN 16657101 . We preset preliminary data from 15 patients with stable COPD (GOLD 2017 criteria) recruited from clinics and pulmonary rehabilitation programs, 10 patients admitted to hospital with ECOPD and 13 healthy CONTROLS (spouses, family members). Spontaneous saliva and sputum were sampled. Metabolites from the samples were extracted using methanol, chloroform and homogenisation using glass beads. The extracts were analysed using the flow infusion electrospray ionisation mass spectrometry (FIE-MS). This yielded a data matrix of over $38 \times 6500$ (metabolites). The results were normalized and analysed using the Metaboanalyst 3.0 which included Principal Component Analysis (PCA), Partial Least Squares Discriminant Analysis (PLS-DA), Analysis of Variation (ANOVA) and T-tests.

\section{Results:}

Multivariate and machine-learning based analyses of the profiles demonstrated that both sputum (Fig. 2) and saliva (Fig.3) provide statistically robust discrimination between healthy controls and both COPD groups, particularly the ECOPD group. The coloured areas represent 95\% confidence intervals for each group and it should be noted that these do not overlap.

Interrogation of the matrices by ANOVA identified the top 20 metabolites which were the source of variation between each patient class. These are displayed as heat maps for sputum (Fig. 4) and saliva (Fig. 5).

The clustering seen in the ECOPD samples further emphasises the distinctiveness of this clinical class.

\section{Discussion:}

The results of this study suggest new technologies testing saliva and sputum metabolomic profiling can reliably differentiate ECOPD from stable COPD and CONTROLS. Further analysis of 150 samples is underway which may permit differentiation of patients into various GOLD categories (ABCD) and determine the influence of antibiotics and steroids on treatment outcomes (ECOPD versus stable COPD within the same patient) from a single sputum or saliva test to enable clinicians to focus on personalized therapeutics. Saliva and sputum samples from exacerbating COPD patients possess distinctive metabolomic signatures.
Fig. 2 - Principal Component Analysis: Analysis of biochemicals in SPUTUM

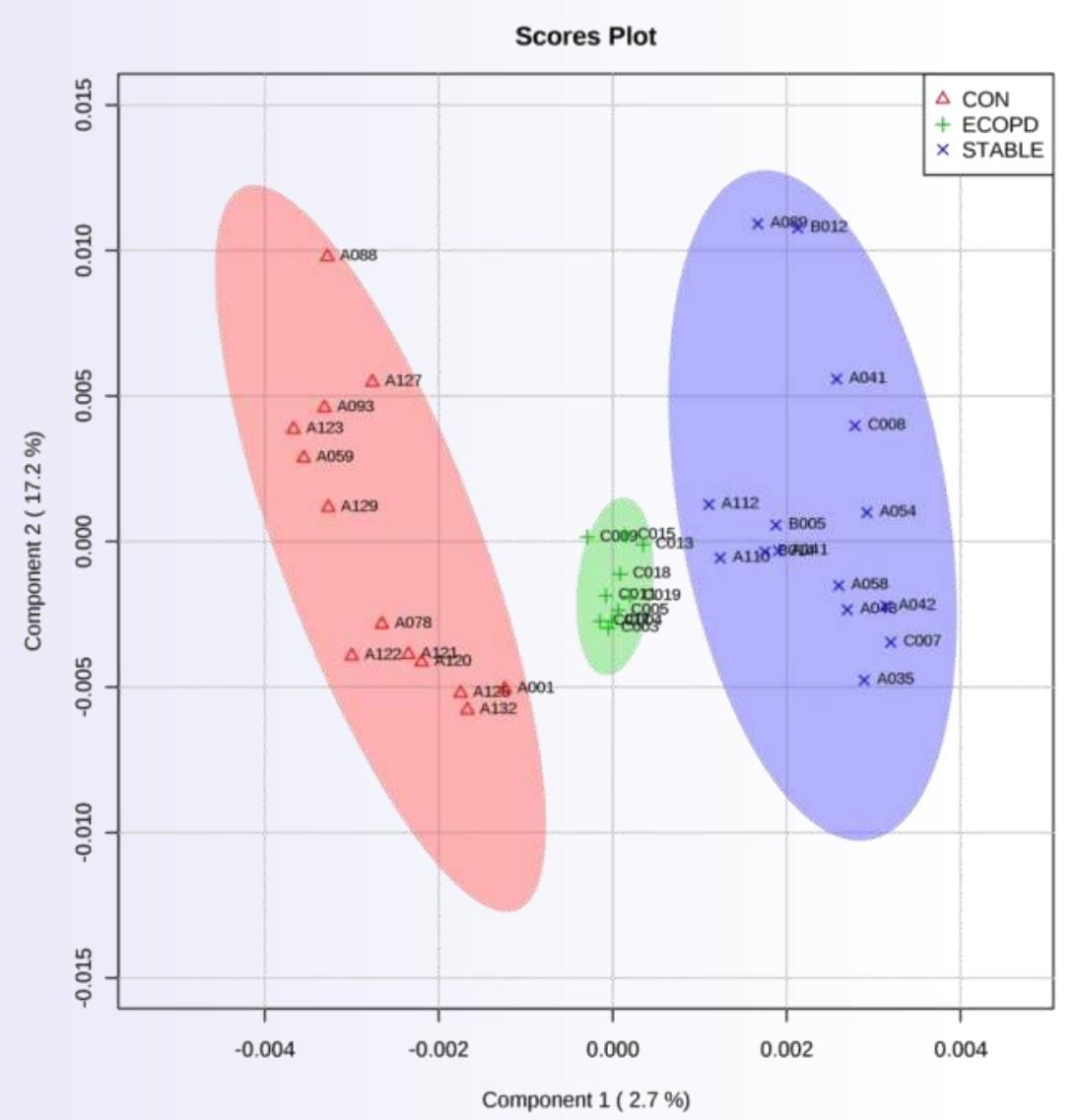

Fig. 4 - Heat Map: Top 20 significant METABOLITES changing in SPUTUM

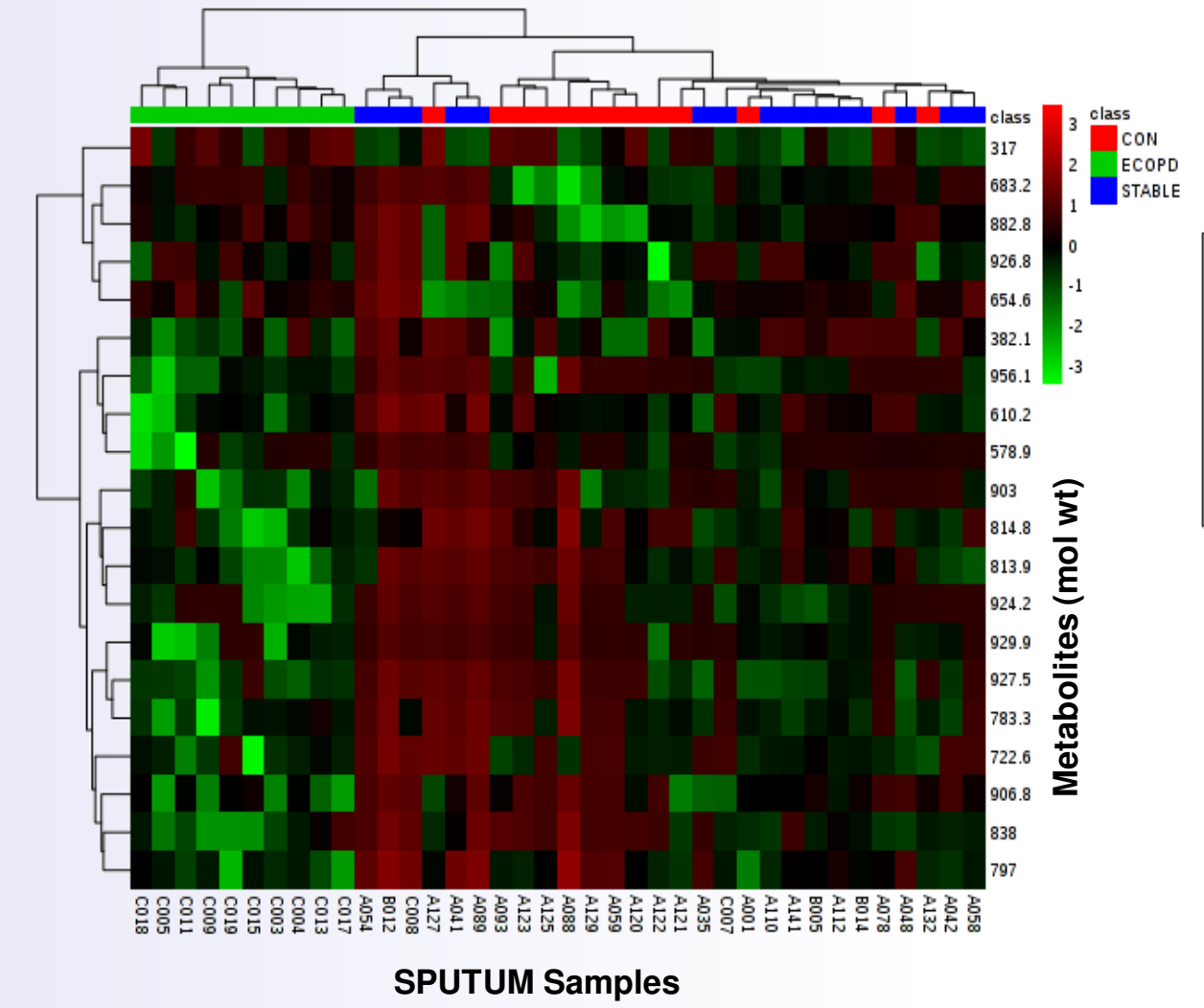

SPUTUM Samples
Fig. 3 - Principal Component Analysis: Analysis of biochemicals in SALIVA

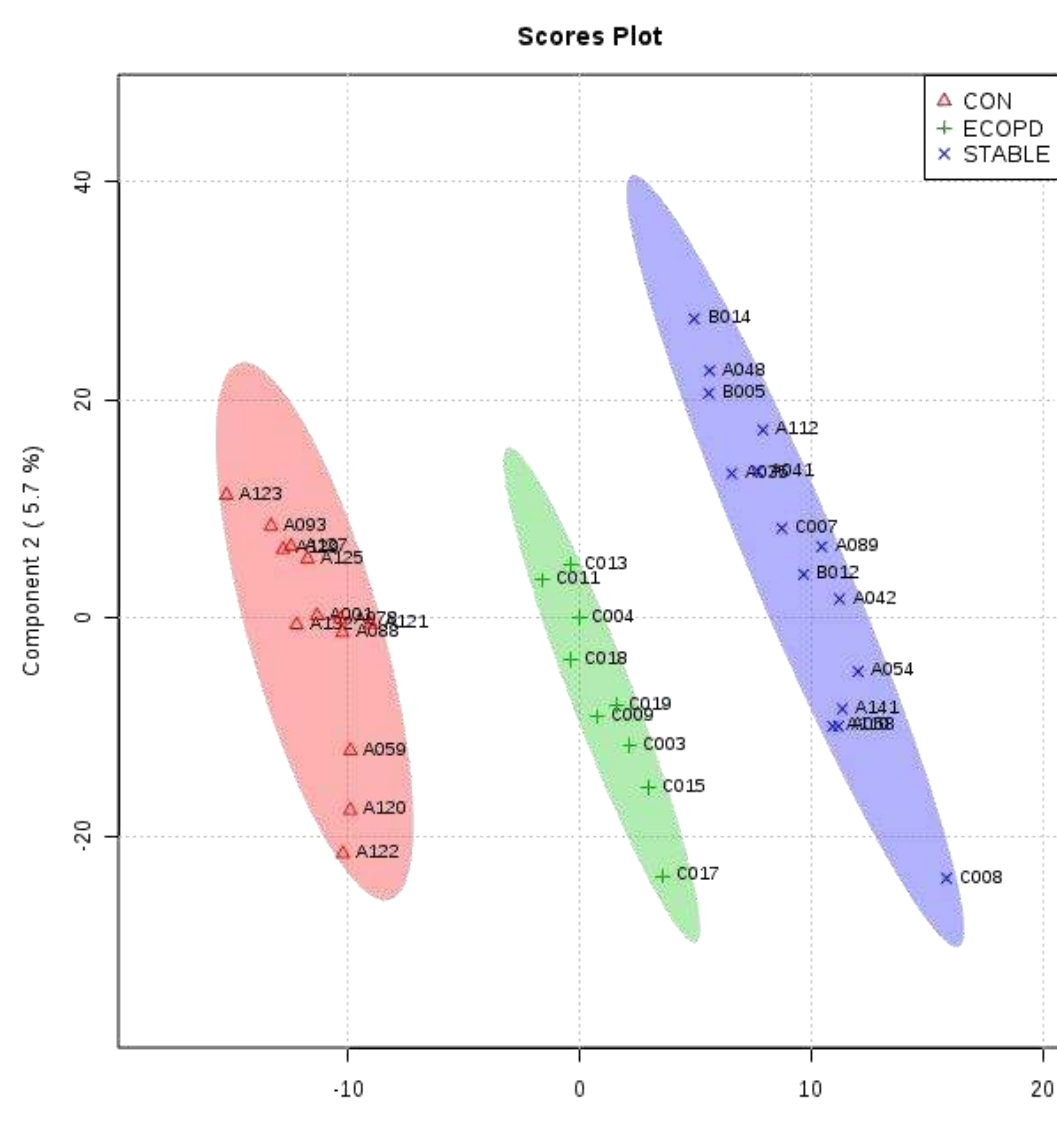

Fig. 5 - Heat Map: Top 20 significant METABOLITES changing in SALIVA

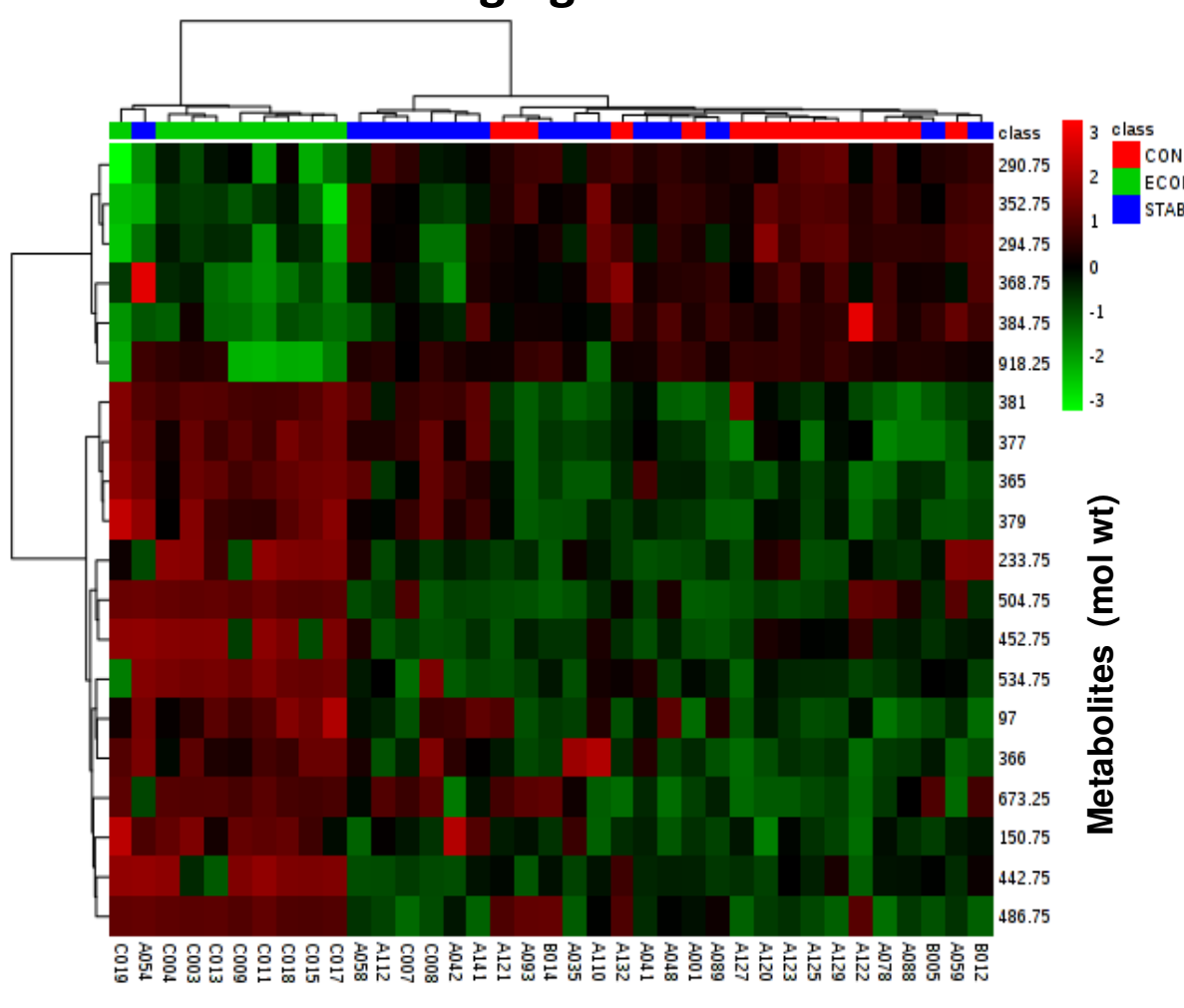

SALIVA Samples 